\title{
Bloom Filters and Compact Hash Codes for Efficient and Distributed Image Retrieval
}

\author{
Andrea Salvi, Simone Ercoli, Marco Bertini and Alberto Del Bimbo \\ MICC \\ Università degli Studi di Firenze \\ Firenze, Italy \\ Email: andreasalvi89@gmail.com,marco.bertini@unifi.it,alberto.delbimbo@unifi.it
}

\begin{abstract}
This paper presents a novel method for efficient image retrieval, based on a simple and effective hashing of CNN features and the use of an indexing structure based on Bloom filters. These filters are used as gatekeepers for the database of image features, allowing to avoid to perform a query if the query features are not stored in the database and speeding up the query process, without affecting retrieval performance. Thanks to the limited memory requirements the system is suitable for mobile applications and distributed databases, associating each filter to a distributed portion of the database (database shard), addressing large scale archives and allowing query parallelization. Experimental validation has been performed on three standard image retrieval datasets, outperforming state-of-the-art hashing methods in terms of precision, while the proposed indexing method obtains a $2 \times$ speedup.
\end{abstract}

Keywords-Nearest neighbor retrieval; hashing; Bloom filter; CNN;

\section{INTRODUCTION}

Content based image retrieval (CBIR) has been an active research topic in computer vision and multimedia in the last decades, and it is still very relevant due to the emergence of social networks and the creation of web-scale image databases. Most of the works have addressed the development of effective visual features, from engineered features like SIFT and GIST to, more recently, learned features such as CNNs [1]. To obtain scalable CBIR systems features are typically compressed or hashed, to reduce their dimensionality and size. However, research on data structures that can efficiently index these descriptors has attracted less attention, and typically simple inverted files (e.g. implemented as hash tables) are used.

In this paper we address the problem of approximate nearest neighbor (ANN) image retrieval proposing a simple and effective data structure that can greatly reduce the need to perform any comparison between the descriptor of the query and those of the database, when the probability of a match is very low. Considering the proverbial problem of finding a needle in a haystack, the proposed system is able to tell when the haystack probably contains no needle and thus the search can be avoided completely.
To achieve this we propose a novel variation of an effective hashing method for CNN descriptors, and use this code to perform ANN retrieval in a database. To perform an immediate rejection of a search that should not return any result we store the hash code in a Bloom filter, i.e. a space efficient probabilistic data structure that is used to test the presence of an element in a set. To the best of our knowledge this is the first time that this data structure has been proposed for image retrieval since, natively, it has no facility to handle approximate queries. We perform extensive experimental validation on three standard datasets, showing how the proposed hashing method improves over state-of-the-art methods, and how the data structure greatly improves computational cost and makes the system suitable for application to mobile devices and distributed image databases.

\section{PREVIOUS WORKS}

Visual features: SIFT descriptors have been successfully used for many years to perform CBIR. Features have been aggregated using Bag-of-Visual-Words and, with improved performance, using VLAD [2] and Fisher Vectors [3].

The recent success of CNNs for image classification tasks has suggested their use also for image retrieval tasks. Babenko et al. [1] have proposed the use of different layers of CNNs as features, compressing them with PCA to reduce their dimensionality, and obtaining results comparable with state-of-the-art approaches based on SIFT and Fisher Vectors. Aggregation of local CNN features using VLAD has been proposed in [4], while Fisher Vectors computed on $\mathrm{CNN}$ features of objectness window proposals have been used in [5].

Hashing: One of the most successful visual feature hashing methods presented in the literature is Product Quantization (PQ), proposed by Jégou et al. [6]. In this method the feature space is decomposed into a Cartesian product of subspaces with lower dimensionality, that are quantized separately. The method has obtained state-of-the-art results on a large scale SIFT and GIST features dataset. The good performance of the Product Quantization method has led to development of several related methods that introduce 
variations and improvements. Norouzi and Fleet [7] have built two variations of k-means (Orthogonal k-means and Cartesian k-means) upon the idea of compositionality of the PQ approach. Ge et al. [8] have improved PQ minimizing quantization distortions w.r.t. space decomposition and quantization codebooks, in their OPQ method; He et al. [9] have approximated the Euclidean distance between codewords in k-means method, proposing an affinity-preserving technique. More recently, Kalantidis and Avrithis [10] have proposed to use a local optimization over a rotation and a space decomposition, applying a parametric solution that assumes a normal distribution, in their vector quantization method (LOPQ).

Most of recent approaches for $\mathrm{CNN}$ features hashing are based on simultaneous learning of image features and hash functions as in the method of Gao et al. [11], that uses visual and label information to learn a relative similarity graph, to reflect more precisely the relationship among training data.

In [12] CNN binary hash codes are learned through a two stage approach: in the first one a supervised pre-training is performed on ImageNet, then in the second phase is added a latent layer to the $\mathrm{CNN}$ network, to learn hash like representations, during a fine tuning process on the target image dataset. Retrieval is performed using a coarse-to-fine approach, based on hash codes (coarse) and FC7 features (fine).

Unsupervised two steps hashing of CNN features has been proposed by Lin et al. [13]. In the first step Stacked Restricted Boltzmann Machines learn binary embedding functions, then fine tuning is performed to retain the metric properties of the original feature space.

Indexing: Typically hashed features are stored in inverted files. A few works have studied other data structures to speed up approximate nearest neighbors. Babenko and Lempitsky [14] have proposed an efficient similarity search method that generalizes the inverted index; the method, called inverted multi-index (Multi-D-ADC), replaces vector quantization inside inverted indices with product quantization, and builds the multi-index as a multi-dimensional table. Ercoli et al. [15] have proposed an hashing method that improves over PQ by performing multiple assignments to k-means centroids, and have stored the hash codes in Marisa Tries to greatly compress their storage.

Bloom filter: Bloom filter and its many variants have received an extremely limited attention from the vision and multimedia community, so far. Inoue and Kise [16] have used Bloomier filters (i.e. an associative array of Bloom filters) to store PCA-SIFT features of an objects dataset more efficiently than using an hash table; they perform object recognition by counting how many features stored in the filters are associated with an object. Bloom filter has been used by Danielsson [17] as feature descriptor for matching keypoints. Similarity of descriptors is evaluated using the "union" operator. Srijan and Jawahar have proposed to use
Bloom filters to store compactly the descriptors of an image, and use the filter as postings of an inverted file index in [18]. More recently Araujo et al. [19] have proposed the use of Bloom filters for query-by-image video retrieval, where video scenes are stored as Fisher-embedded local features in Bloom filters.

\section{The Proposed Method}

In the proposed approach, differently from [11], we learn a vector quantizer separately from the CNN features, so to easily replace different and pre-trained CNN networks for feature extraction, without need of retraining. Moreover, we propose to include Bloom filters into feature indexing structures to improve the speed of queries. Bloom filters act as gatekeepers that rule out immediately, with a very limited memory cost, if a query should be completely performed or if it can be avoided. The proposed data structure is very suitable for mobile and distributed applications.

\section{A. Quantization Algorithm}

The proposed approach is a variation of [15], which is an efficient method for mobile visual search based on a multiple assignment $\mathrm{k}$-means hashing schema (multi-kmeans) that obtained very good results, compared to PQ, on the BIGANN dataset.

The first step of the method consists in learning a standard k-means dictionary with a small number of centroids (to maintain a low computational cost). Each centroid is associated to a bit of the hash code, that has thus length equal to the number of centroids. The bit is set to 1 if the feature is assigned to the centroid, 0 otherwise. A feature can be assigned to more than one centroid, and it is assigned to it if the distance from the centroid is less than the mean distance from all the centroids (Figure 1, top). Instead, in this work we select a fixed number $N$ of distances and we set to 1 all the bits associated to the smaller $N$ distances (Figure 1, bottom). In the following we refer to this method as MINx. This change has proven to be more efficient when coding CNN feature descriptors, that were used in the experiments.

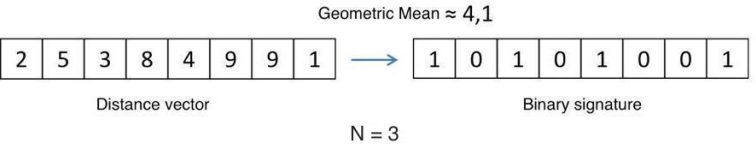

$$
\begin{aligned}
& \begin{array}{|c|c|c|c|c|c|c|c|}
\hline 2 & 5 & 3 & 8 & 4 & 9 & 9 & 1
\end{array} \rightarrow \begin{array}{|c|c|c|c|c|c|c|c|}
\hline 1 & 0 & 1 & 0 & 0 & 0 & 0 & 1 \\
\hline \multicolumn{10}{c|}{\text { Dinary signature }}
\end{array}
\end{aligned}
$$

Figure 1. Binarization examples with a distance vector of 8 elements: (top) geometric mean (MEAN method); (bottom) smaller distances $N=3$ (MINx method).

Approximate nearest neighbor retrieval of image descriptors is performed in two steps: in the first step is performed an exhaustive search over the binary codes using Hamming distances, to reduce negative effects of quantization errors. 
All the binary codes with Hamming distance below a threshold are selected. In the second step the candidate neighbors are re-ranked according to the distance computed using the full feature vector. In this case the distance is computed using cosine distance, that proved to be more effective than $L_{2}$ during the experiments.

\section{B. Bloom Filter Algorithm}

To improve search of feature vectors we also introduce the use of Bloom filters [20]. Typically this type of structures are used to speed up the answers in a key-based storage system (Figure 2).

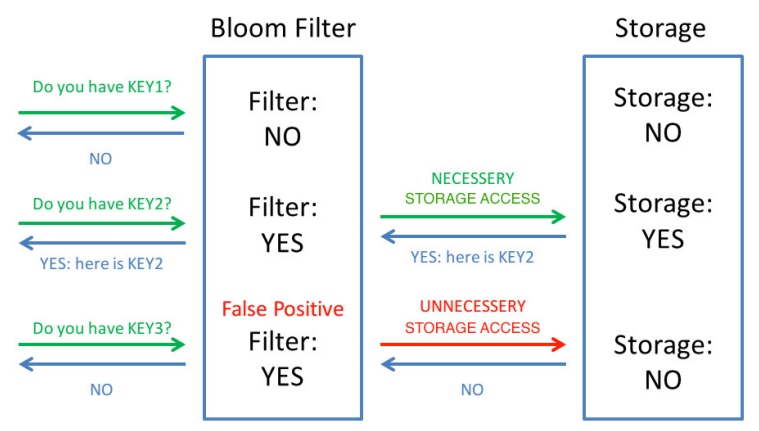

Figure 2. Memory accesses with Bloom filters

A Bloom filter is an efficient probabilistic data structure used to test if an element belongs to a set or not. This structure works with binary signatures, and can provide false positive response but not false negative and more elements are inserted into the structure and more high is the probability to obtain a false positive. To insert an element inside a Bloom filter we need to define $k$ hash functions which locate $k$ positions inside the array, setting them to 1 .

To check the presence of an element inside a Bloom filter we to compute the $k$ hash functions over the element and check the related positions inside the array. If at least one bit of these positions is equal to 0 it means that the element is not present inside the array; if all the checked bits are equal to 1 it means that either the element is inside the array or we have a false positive. We used the method of [21] to create the $k$ functions from just two hash functions, that in our implementation were based on MurmurHash3.

A useful property of Bloom filter is that we can measure the presence of a false positive with probability:

$$
\left(1-e^{-k n / m}\right)^{k}=(1-p)^{k}=\epsilon
$$

where $m$ is the bit size of the array, $n$ is the number of inserted items, $p$ is the probability that one position of the array is equal to 0 , and $k$ is the number of hash functions (that should be perfectly random). We can obtain the optimal value $k$, given $n$ and $m$, which minimizes false positive probability. Typically $m=O(n)$ it is a good compromise.
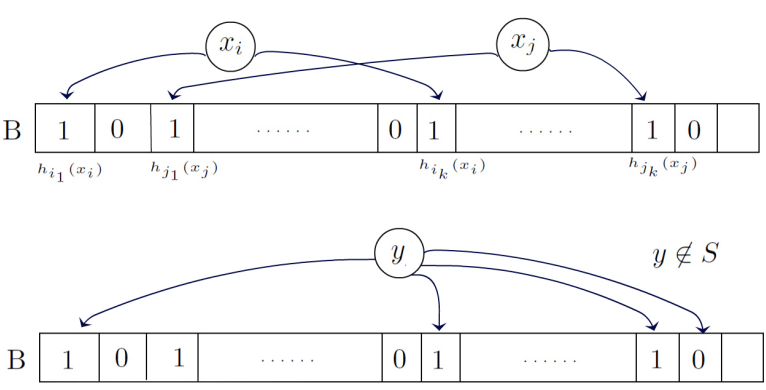

Figure 3. Example Bloom filter: (top) Insertion, (bottom) Search

This property makes the Bloom filters interesting for exact retrieval; to handle the case of approximate testing, two variations called Distance-Sensitive Bloom filter (DSBF) [22] and Locality-Sensitive Bloom Filters (LSBF) [23], have been proposed, where LSH functions are used as $k$ hash functions. In our approach, storing in the Bloom filter, using the $k$ random hash functions, the hash codes designed for ANN, as those of Sect. III-A, results in a data structure that is similar, from a practical point of view, to DSBFs and LSBFs. In this approach the ANN hash codes are required to reduce the false negatives; this avoids the need to use a multi-probe approach as in [23]. Experimental results show that the ANN signature is effective in avoiding the issue (see Tab. II).

\section{Retrieval System}

Our proposed retrieval system merges the methods introduced in III-A and III-B. Regarding visual feature hashing we have applied the proposed method to CNNs features. The system (Figure 4) uses a coarse-to-fine approach: in the coarse phase hashes are used to select a subset of images that are re-ranked in the fine phase.

More in detail in the initial phase descriptors are extracted from base images, binarized following one of the methods introduced in III-A and saved inside a data structure composed by a set of inverted files of hashes implementing an horizontal partition of data (allowing to distribute the database as "shard"), each one guarded by a Bloom filter. The hash code is also added to the Bloom filter of the corresponding inverted file.

During the search phase we extract the CNN descriptor from query images, compute the hash code, and check the presence of the hash in the Bloom filters, each of which guard a subset of the database (shard). If one of this Bloom filters gives a positive response (this means that we have a positive or a false positive match), all the hash codes within an Hamming distance threshold are used to select the full feature vector. This provides a great speedup in the approximate nearest neighbor retrieval since we consider only descriptors from base coded by a Bloom filter, and below the Hamming threshold value. For each resulting original CNN descriptor we compute the distance and we rearrange results to obtain a ranked list of vectors. 


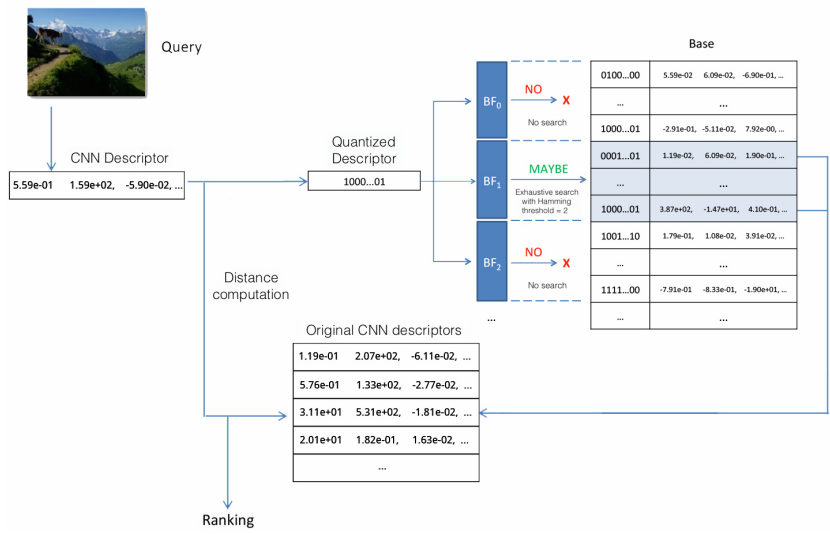

Figure 4. System overview: a CNN descriptor is hashed to 64 bits, used to query a sharded database. Each database shard is accessed through a Bloom filter that contains the hashes of the CNN descriptors stored in the DB. If the Hamming distance between the query hash and the DB hash is less than a threshold the full CNN descriptors are selected to re-rank the final list.

\section{EXPERIMENTAL RESULTS}

\section{A. Datasets and Configurations}

We tested our system using three standard dataset: INRIA Holidays [24], Oxford 5K [25] and Paris 6K [26]. We used the query images and ground truth provided for each dataset, adding 100,000 distractor images from Flickr 100 [27]. When testing on a dataset training is performed using the other two datasets. Features have been hashed to 64 bits binary codes, a length that has proved to be the best compromise between compactness and representativeness. Other parameters used for hashing were:

- number of $N$ nearest distances used in the hash code computation $(N \in\{6,10,16,32,40\})$;

- Hamming distance threshold $\in\{2,4,6,10,16,30\}$.

For the sake of brevity, in the following we report only the best combinations. For the evaluation we used the Mean Average Precision (MAP) metric. The CNN features used in the following experiments have been extracted using the $1024 d$ average pooling layer of GoogLeNet [28], that in initial experiments has proven to be more effective than the FC7 layer of VGG [29] used in [5].

\section{B. Results}

In the first experiment we evaluate the effects of the method parameters, comparing the proposed hashing approach (MINx) with the original method of [15] (MEAN), a baseline that uses no hashing, and many state-of-theart methods, among which the recent UTH method [13]. Although, several of these methods have been originally tested on engineered features, in this case the results have been obtained hashing $\mathrm{CNN}$ features; figures reported have been obtained from [13], using a VGG convnet. The best combinations of MINx are reported, compared on the three datasets in terms of MAP. As expected the uncompressed features perform better, but the MIN6 setup, with an Hamming distance $\geq 6$ has comparable results, and greatly outperforms any state-of-the-art hashing method. A more detailed evaluation of the parameters of the proposed hashing method is reported in Figure 5 for the INRIA Holidays dataset. Time results in seconds, for INRIA Holidays dataset, are reported in Figure 6 . A $2 \times-10 \times$ speedup can be obtained with Hamming distances between 6 and 10. Similar results have been obtained on Oxford $5 \mathrm{~K}$ and Paris $6 \mathrm{~K}$ datasets.

Table I

MAP RESUlTS ON HOLIDAYS, OXFORD 5K AND PARIS 6K DATASETS. THE PROPOSED MINX METHOD OUTPERFORMS ALL THE CURRENT STATE-OF-THE-ART METHODS. ALL HASHES ARE 64 BIT LONG AND HAVE BEEN COMPUTED ON CNN DESCRIPTORS.

\begin{tabular}{|c|c|c|c|}
\hline Method & Holidays & Oxford 5K & Paris 6K \\
\hline ITQ [30] & 53.68 & 23.00 & - \\
\hline BPBC [31] & 38.10 & 22.51 & - \\
\hline PCAHash [30] & 52.80 & 23.90 & - \\
\hline LSH [32] & 43.08 & 23.91 & - \\
\hline SKLSH [33] & 24.09 & 13.39 & - \\
\hline SH [34] & 52.22 & 23.24 & - \\
\hline SRBM [35] & 51.58 & 21.23 & - \\
\hline UTH [13] & 57.10 & 24.00 & - \\
\hline \hline $\begin{array}{c}\text { MIN6 } \\
\text { Thr. 10 }\end{array}$ & $\mathbf{7 5 . 6 2}$ & $\mathbf{4 6 . 0 3}$ & 67.57 \\
\hline $\begin{array}{c}\text { MIN6 } \\
\text { Thr. 16 }\end{array}$ & $\mathbf{7 5 . 6 3}$ & $\mathbf{4 6 . 0 6}$ & $\mathbf{6 7 . 8 4}$ \\
\hline $\begin{array}{c}\text { MIN10 } \\
\text { Thr. 10 }\end{array}$ & 74.33 & 45.66 & 60.84 \\
\hline $\begin{array}{c}\text { MIN10 } \\
\text { Thr. 16 }\end{array}$ & $\mathbf{7 5 . 6 3}$ & 46.04 & 67.70 \\
\hline $\begin{array}{c}\text { MEAN [15] } \\
\text { Thr. 10 }\end{array}$ & 68.26 & 36.22 & 48.02 \\
\hline Baseline & 75.63 & 46.06 & 67.84 \\
\hline
\end{tabular}

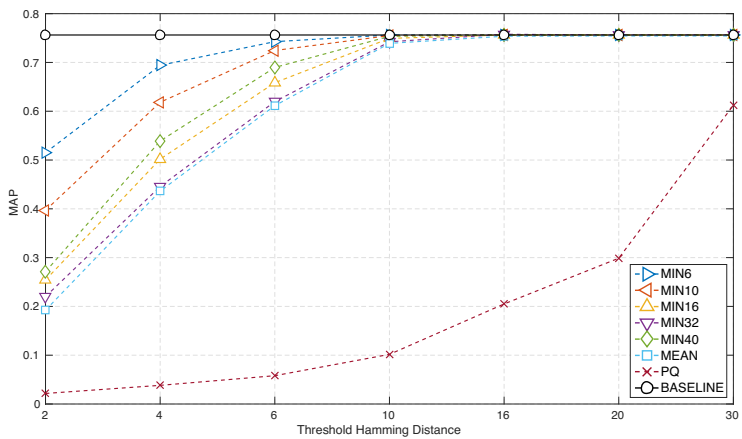

Figure 5. INRIA Holidays: MAP results on Holidays dataset for the proposed MIN method, with different thresholds, compared to the MEAN [15] and Product Quantization [6] methods, and baseline without hashing.

In the second experiment we evaluate a use case in which a database of images is queried with a large number of images that do not belong to it. Hash codes have been 


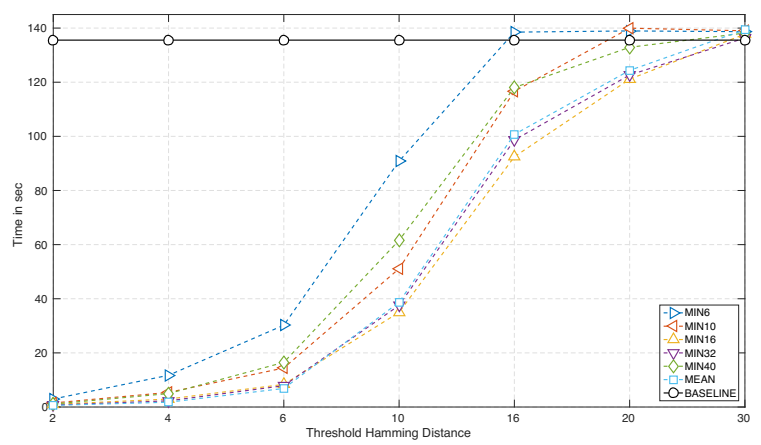

Figure 6. INRIA Holidays: time comparison for the MINx method, the MEAN [15] method and baseline without hashing.

computed with different variants of the proposed hashing method. The database contains the Paris $6 \mathrm{~K}$ images, and it is queried with all the query images of Paris $6 \mathrm{~K}$ and all the 100,000 distractor images. A different number of Bloom filters, with different sizes is tested and compared against a baseline that does not use any Bloom filter. MAP values (computed on Paris queries) and query time in seconds are reported in Tab. II (MAP in the first row and time in the second). The speedup obtained is about $2 \times$ since a large number of distractor queries are immediately stopped by the system; the slight increase in MAP is due to the beneficial effect of elimination of some false positives of the Paris $6 \mathrm{~K}$ images, that do not result in retrieving wrong dataset images.

Table II

MAP+TIME (SECS.) OBTAINED ON PARIS 6K WITH THE PROPOSED SYSTEM WITH DIFFERENT NUMBERS OF BLOOM FILTERS (1, 2 AND 5) AND WITH A BASELINE WITHOUT FILTERS. $2 n$ AND $5 n$ ARE THE SIZE OF THE FILTERS, WHERE $n$ IS THE NUMBER OF STORED ELEMENTS. Thr. IS THE MAX. HAMMING DISTANCE USED FOR HASH CODE RETRIEVAL.

\begin{tabular}{|c|c|c|c|c|c|c|c|}
\hline & No & \multicolumn{2}{|c|}{ 1 BF } & \multicolumn{2}{c|}{ 2 BF } & \multicolumn{2}{c|}{ 5 BF } \\
\cline { 3 - 8 } & BF & 2n & 5n & 2n & 5n & 2n & 5n \\
\hline MIN6 & 67.57 & 67.57 & 67.57 & 67.94 & $\mathbf{7 0 . 9 6}$ & 67.53 & 68.21 \\
Thr. 10 & 460.31 & 242.72 & 173.46 & 311.26 & $\mathbf{2 0 5 . 0 8}$ & 382.14 & 366.61 \\
\hline MIN10 & 67.70 & 67.69 & 67.69 & 68.37 & 68.46 & 67.66 & 66.70 \\
Thr. 16 & 553.79 & 307.46 & 174.53 & 459.74 & 272.31 & 543.65 & 430.06 \\
\hline
\end{tabular}

In the third experiment we evaluate a more challenging and large scale experiment: three datasets composed by distractor images and Holidays, Paris $6 \mathrm{~K}$ and Oxford $5 \mathrm{~K}$ images are built and stored in the proposed data structure. The standard dataset query images are then used to query the system. In this case we have used 10 filters to "shard" the database that, thus, can be distributed. Tab. III reports the results in terms of MAP and time (secs.). For the sake of space we report only results for MIN6 and Hamming threshold 10. Using the proposed method results in speed improvement of $2 \times$ while improving MAP, except the Holidays dataset that only improves speed. The size of each Bloom filter is $\sim 6-62 \mathrm{~KB}$, allowing the use of the method in a mobile environment, by distributing the Bloom filters to the mobile devices and maintaining the shards of the database on the backend.

Table III

MAP+TIME (SECS.) OBTAINED ON PARIS 6K, OXFORD 5K AND HOLIDAYS WITH THE PROPOSED SYSTEM WITH 10 BLOOM FILTERS OF VARYING SIZE AND WITH A BASELINE WITHOUT FILTERS. THE DATABASE CONTAINS 100,000 DISTRACTOR + THE DATABASE IMAGES OF EACH DATASET.

\begin{tabular}{|c|r|r|r|}
\hline \# BF & Paris 6K & Oxford 5K & Holidays \\
\hline \hline No BF & 58.52 & 42.05 & $\mathbf{5 9 . 5 6}$ \\
& 3.35 & 2.35 & 56.71 \\
\hline $10 \mathrm{BF}$ & 59.44 & 41.45 & 52.15 \\
$10 \mathrm{n}$ & 2.66 & 1.95 & 47.21 \\
\hline $10 \mathrm{BF}$ & 61.21 & 42.01 & 42.36 \\
$20 \mathrm{n}$ & 1.93 & 1.53 & 36.36 \\
\hline $10 \mathrm{BF}$ & 62.82 & $\mathbf{4 2 . 2 9}$ & 39.26 \\
$30 \mathrm{n}$ & 1.43 & 1.25 & 31.37 \\
\hline $10 \mathrm{BF}$ & $\mathbf{6 3 . 5 2}$ & $\mathbf{4 2 . 2 4}$ & 34.29 \\
$50 \mathrm{n}$ & $\mathbf{1 . 2 1}$ & $\mathbf{1 . 1 1}$ & $\mathbf{2 6 . 5 9}$ \\
\hline
\end{tabular}

\section{CONCLUSIONS}

In this paper we have presented a simple and effective method for CNN feature hashing that outperforms current state-of-the-art methods on standard datasets; the proposed method is robust with respect to its two parameters, and has also the added value of being simple so that it can be easily re-implemented.

A novel indexing structure, where Bloom filters are used as gatekeepers to inverted files storing the hash codes, results in a $2 \times$ speedup for ANN, without loss in MAP. The indexing structure allows to distribute the inverted files used to store the hashes and descriptors of images as database shards. Given the very compact size of the Bloom filters it is also viable to distribute these indexes to clients running on mobile devices, so to reduce the cost of querying remote databases.

\section{ACKNOWLEDGMENTS}

This work is partially supported by the "Social Museum and Smart Tourism" project (CTN01_00034_231545).

This research is based upon work supported [in part] by the Office of the Director of National Intelligence (ODNI), Intelligence Advanced Research Projects Activity (IARPA), via IARPA contract number 2014-14071600011. The views and conclusions contained herein are those of the authors and should not be interpreted as necessarily representing the official policies or endorsements, either expressed or implied, of ODNI, IARPA, or the U.S. Government. The U.S. Government is authorized to reproduce and distribute reprints for Governmental purpose notwithstanding any copyright annotation thereon.

\section{REFERENCES}

[1] A. Babenko, A. Slesarev, A. Chigorin, and V. Lempitsky, "Neural codes for image retrieval," in Proc. of ECCV, 2014.

[2] H. Jegou, F. Perronnin, M. Douze, J. Sanchez, P. Perez, and C. Schmid, "Aggregating local descriptors into compact codes," IEEE Transactions on Pattern Analysis and Machine Intelligence, vol. 34, no. 9, pp. 1704-1716, 2012. 
[3] J. Sánchez, F. Perronnin, T. Mensink, and J. Verbeek, "Image classification with the Fisher vector: Theory and practice," International Journal of Computer Vision, vol. 105, no. 3, pp. 222-245, 2013.

[4] J. Yue-Hei Ng, F. Yang, and L. S. Davis, "Exploiting local features from deep networks for image retrieval," in Proc. of CVPR Workshops, June 2015.

[5] T. Uricchio, M. Bertini, L. Seidenari, and A. Del Bimbo, "Fisher encoded convolutional bag-of-windows for efficient image retrieval and social image tagging," in Proc. of ICCV Workshops, 2015.

[6] H. Jégou, M. Douze, and C. Schmid, "Product quantization for nearest neighbor search," IEEE Transactions on Pattern Analysis and Machine Intelligence, vol. 33, no. 1, pp. 117128, 2011.

[7] M. Norouzi and D. Fleet, "Cartesian k-means," in Proc. of CVPR, 2013.

[8] T. Ge, K. He, Q. Ke, and J. Sun, "Optimized product quantization for approximate nearest neighbor search," in Proc. of CVPR, 2013.

[9] K. He, F. Wen, and J. Sun, "K-means hashing: An affinitypreserving quantization method for learning binary compact codes," in Proc. of CVPR, 2013.

[10] Y. Kalantidis and Y. Avrithis, "Locally optimized product quantization for approximate nearest neighbor search," in Proc. of CVPR, 2014, pp. 2329-2336.

[11] L. Gao, J. Song, F. Zou, D. Zhang, and J. Shao, "Scalable multimedia retrieval by deep learning hashing with relative similarity learning," in Proc. of ACM MM, 2015.

[12] K. Lin, H.-F. Yang, J.-H. Hsiao, and C.-S. Chen, "Deep learning of binary hash codes for fast image retrieval," in Proc. of CVPR, 2015.

[13] J. Lin, O. Morère, J. Petta, V. Chandrasekhar, and A. Veillard, "Tiny descriptors for image retrieval with unsupervised triplet hashing," in Proc. of DCC, 2016.

[14] A. Babenko and V. Lempitsky, "The inverted multi-index," in Proc. of CVPR, 2012.

[15] S. Ercoli, M. Bertini, and A. Del Bimbo, "Compact hash codes and data structures for efficient mobile visual search," in Proc. of ICME Workshops, 2015.

[16] K. Inoue and K. Kise, "Compressed representation of feature vectors using a Bloomier filter and its application to specific object recognition," in Proc. of ICCV Workshops, 2009.

[17] O. Danielsson, "Category-sensitive hashing and Bloom filter based descriptors for online keypoint recognition," in Proc. of SCIA, 2015.

[18] K. Srijan and C. V. Jawahar, "Towards exhaustive pairwise matching in large image collections," in Proc. of ECCV, 2012.
[19] A. Araujo, J. Chaves, H. Lakshman, R. Angst, and B. Girod, "Large-scale query-by-image video retrieval using bloom filters," arXiv preprint arXiv:1604.07939, 2016.

[20] B. H. Bloom, "Space/time trade-offs in hash coding with allowable errors," Communications of the ACM, vol. 13, no. 7, pp. 422-426, 1970.

[21] A. Kirsch and M. Mitzenmacher, "Less hashing, same performance: Building a better Bloom filter," Random Structures \& Algorithms, vol. 33, no. 2, pp. 187-218, 2008.

[22] —_, "Distance-sensitive Bloom filters," in Proc. of ALENEX, 2006.

[23] Y. Hua, B. Xiao, B. Veeravalli, and D. Feng, "Localitysensitive Bloom filter for approximate membership query," IEEE Transactions on Computers, vol. 61, no. 6, pp. 817830,2012

[24] H. Jegou, M. Douze, and C. Schmid, "Hamming embedding and weak geometric consistency for large scale image search," in Proc. of ECCV, 2008.

[25] J. Philbin, O. Chum, M. Isard, J. Sivic, and A. Zisserman, "Object retrieval with large vocabularies and fast spatial matching," in Proc. of CVPR, 2007.

[26] —, "Lost in quantization: Improving particular object retrieval in large scale image databases," in Proc. of CVPR, 2008.

[27] _ , "Object retrieval with large vocabularies and fast spatial matching," in Proc. of CVPR, 2007.

[28] C. Szegedy, W. Liu, Y. Jia, P. Sermanet, S. Reed, D. Anguelov, D. Erhan, V. Vanhoucke, and A. Rabinovich, "Going deeper with convolutions," in Proc. of CVPR, June 2015.

[29] K. Simonyan and A. Zisserman, "Very deep convolutional networks for large-scale image recognition," in Proc. of ICLR, 2015.

[30] Y. Gong and S. Lazebnik, "Iterative quantization: A procrustean approach to learning binary codes," in Proc. of CVPR, 2011.

[31] Y. Gong, S. Kumar, H. Rowley, and S. Lazebnik, "Learning binary codes for high-dimensional data using bilinear projections," in Proc. of CVPR, 2013.

[32] M. Datar, N. Immorlica, P. Indyk, and V. S. Mirrokni, "Locality-sensitive hashing scheme based on p-stable distributions," in Proc. of SoCG, 2004

[33] M. Raginsky and S. Lazebnik, "Locality-sensitive binary codes from shift-invariant kernels," in Proc. of NIPS, 2009, pp. 1509-1517.

[34] Y. Weiss, A. Torralba, and R. Fergus, "Spectral hashing," in Proc. of NIPS, 2009

[35] V. Chandrasekhar, J. Lin, O. Morere, A. Veillard, and H. Goh, "Compact global descriptors for visual search," in Proc. of DCC, 2015 . 